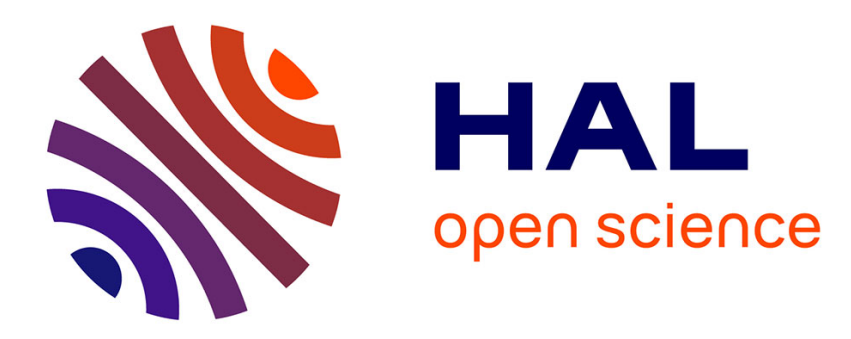

\title{
L'horizon transnational d'une famille tunisienne élargie
}

Hassan Boubakri, Sylvie Mazzella

\section{To cite this version:}

Hassan Boubakri, Sylvie Mazzella. L'horizon transnational d'une famille tunisienne élargie. Autrepart

- Revue de sciences sociales au Sud, 2011, 57-58 (1), pp.111-126. 10.3917/autr.057.0111 . hal01105229

\section{HAL Id: hal-01105229 \\ https://hal.science/hal-01105229}

Submitted on 16 Oct 2015

HAL is a multi-disciplinary open access archive for the deposit and dissemination of scientific research documents, whether they are published or not. The documents may come from teaching and research institutions in France or abroad, or from public or private research centers.
L'archive ouverte pluridisciplinaire HAL, est destinée au dépôt et à la diffusion de documents scientifiques de niveau recherche, publiés ou non, émanant des établissements d'enseignement et de recherche français ou étrangers, des laboratoires publics ou privés. 
HASSAN BOUBAKRI, SYLVIE MAZZELLA, L'horizon transnational d'une famille tunisienne élargie

La question du regroupement familial dépasse de beaucoup la seule problématique de l'intégration dans le débat juridico-politique français, autour du droit ou non au regroupement partiel des familles et de la capacité de la France à enraciner les populations étrangères qui travaillent depuis longtemps sur son sol. Quand elle n'est pas considérée du simple point de vue juridique français, cette question peut être un critère pertinent d'analyse de la famille transnationale élargie. À partir d'une enquête monographique auprès d'une famille tunisienne constituée de sept familles nucléaires, originaire de la zone rurale de Ghoumrassen (région de Tataouine dans le Sud-est tunisien), et dont les membres sont répartis entre Marseille, Tunis et Ghoumrassen, les auteurs cherchent à souligner diverses configurations de mobilité. L'analyse veut démontrer que ces configurations plurielles au sein d'un même clan familial sont liées aux différentes recompositions du projet migratoire «des pères de familles pionniers ». S'ils ont vécu cinquante ans plus tôt une même destinée migratoire, l'expérience transnationale de ces pères révèle en fin de compte des bifurcations professionnelles et familiales divergentes et n'est pas sans effets sur la seconde génération.

Mots-clés : transnationalisme - Tunisie - regroupement familial - intégration - dynamique intergénérationnelle.

\section{HASSAN BOUBAKRI, SYLVIE MAZZELLA, The transnational horizon of an extended Tunisian family}

The issue of family reunification goes way beyond the issue of integration within the context of the French legal and political debate, focusing on the right of partial family reunification and the ability of France to enable the foreign populations who have been working on French soil for a long time to take root. When this issue is not considered from the mere French legal angle, it can be a relevant criterion of analysis as regards the transnational extended family. Based on a monographic survey of a Tunisian family composed of seven nuclear families from the rural area of Ghoumrassen (region of Tataouine, in the South-East of Tunisia), whose members are divided between Marseille, Tunis and Ghoumrassen, the authors seek to underline various mobility patterns. The analysis aims at showing that these different patterns within a same family clan are linked to the different redefinitions of the migratory project of the "pioneer heads of family ». Even if fifty years ago they experienced a similar migratory path, the transnational experience of these fathers ultimately reveals diverging professional and family paths which affect the second generation.

Keywords: transnationalism - Tunisia - family - integration - intergenerational dynamics

Titre courant pair : Hassan Boubakri, Sylvie Mazzella

Titre courant impair: L'horizon transnational d'une famille tunisienne élargie 


\title{
L'HORIZON TRANSNATIONAL D'UNE FAMILLE TUNISIENNE ELARGIE
}

\author{
Hassan Boubakri*, Sylvie Mazzella**
}

À partir du vécu quotidien du regroupement familial d'une famille à cheval entre deux rives, est explorée la construction de l'horizon transnational d'une famille tunisienne élargie, ou plutôt l'élargissement de l'horizon d'une famille tunisienne transnationale.

L'article analyse un clan constitué de sept familles nucléaires, que nous désignerons par la lettre C., originaire de la zone de Ghoumrassen (région rurale de Tataouine dans le Sud-est tunisien), et dont les membres sont répartis entre Marseille, Tunis et Ghoumrassen. Il s'inscrit dans un champ de recherche francophone qui explore depuis les années 1990 la question de l'installation durable de la famille immigrée entre plusieurs espaces nationaux [Quiminal, 1997 ; Streiff-Fénart, 1999 ; Gotman, 1999 ; Bensalah, 1994]. Ce domaine de recherche se développe au moment où des travaux anglo-saxons sur le transnationalisme nuancent un certain nombre de paradigmes en sociologie de l'immigration, notamment l'idée de rupture entre pays d'origine et pays d'accueil. Cette notion représente alors un défi pour la redéfinition et la compréhension d'institutions infranationales telles que la famille [parmi des études récentes : Levitt et Click-Schiller, 2004 ; Caglar, Glick Shiller, Guldbrandsen, 2006 ; Le Gall 2005].

Les chercheurs décrivent moins des entités opposées entre des modèles familiaux traditionnels et ceux de la société d'accueil, que deux ensembles liés au sein desquels les migrants puisent alternativement des ressources matérielles et symboliques en fonction de leurs stratégies propres [Delcroix, Missaoui, 2005]. L'ensemble de ces études ouvre un questionnement encore en friche sur les effets même du processus transnational sur des membres du groupe familial: sur les attentes, et en retour, sur le type d'obligations, que les individus ont à l'endroit de parents géographiquement éloignés, et qui concourent au sentiment d'unité familiale. Ce champ de recherche peut aussi sans doute aider à mieux comprendre le rôle de la famille entre deux types de cadres : celui du dispositif migratoire établi par des générations de migrants entre deux pays, et le cadre législatif évolutif qui les relie.

L'étude revient brièvement sur l'histoire de l'immigration en France de pères commerçants tunisiens, pour souligner dans un second temps le vécu de la procédure de regroupement familial par la famille élargie, et la diversité des formes de mobilités pendulaires qui en découle. Elle analyse enfin quelques réponses apportées par la seconde génération aux effets d'une expérience transnationale « héritée ».

Cette recherche s'efforce ainsi d'éclairer une forme de transnationalisme par le bas qui restructure - dans la durée et dans la pratique sociale ancrée dans la bi- (voire la tri-) polarité - les liens de parenté entre générations, entre hommes et femmes, entre parents et enfants, entre mobiles et non mobiles. Elle espère illustrer ce que Peggy Levitt et Nina Glick-Schiller nomment «un champ social transnational » [2004].

\section{Une enquête de terrain multi-sites}

\footnotetext{
* Professeur à l'Université de Sousse en Tunisie à la faculté des Lettres et des Sciences Humaines, Département de Géographie.

** Sociologue, Chargée de recherche au CNRS, Laboratoire Méditerranéen de Sociologie (LAMES), MMSH, Aix-enProvence.
} 
Les différents cercles de la famille $\mathrm{C}$. sont issus de la même lignée patrilinéaire à la tête de laquelle se trouve leur arrière grand-père Ben R'houma, dont les deux fils (Salem et Mohamed) sont les ascendants respectifs de chacun des deux groupes de cousins éloignés (de troisième degré) interrogés avec leurs familles : soit un premier groupe composé de trois frères (Belgacem, M'Hemed et Mohcen), et un second de quatre frères (Salem décédé, Haj M'hemed, Moktar, et R'houma), âgés de 55 à 65 ans, ainsi que leurs descendants (trente-sept personnes) et collatéraux (sept personnes). Parmi eux, quarante personnes ont été interrogées sur leur récit biographique et leur trajectoire socioprofessionnelle à partir d'entretiens répétés en trois sites (Marseille, Tunis et Ghoumrassen) sur une durée d'enquête de trois ans. Les entretiens (individuels et collectifs) se sont déroulés en français et en tunisien, et il n'était pas rare de devoir parler les deux langues au cours d'un même entretien quand ce dernier réunissait plusieurs membres d'une même famille. Ils ont été effectués soit au domicile des enquêtés à Ghoumrassen et à Marseille, soit dans leurs commerces à Tunis et à Marseille, soit sur leurs terres agricoles à Ghoumrassen. La circulation entre les trois sites a permis de mieux recouper les informations sur les liens de parenté et sur les trajectoires socioprofessionnelles, de combler des zones d'ombre, de saisir les oublis des uns, les non-dits des autres, et de s'entretenir auprès des plus mobiles comme auprès de ceux et celles qui ne se déplacent pas dans le groupe. Cela a aussi permis d'observer et de mieux contextualiser les informations recueillies par entretien (composition de la famille aux différents domiciles, caractéristiques du logement, type et organisation de l'activité commerciale), et d'établir une plus grande familiarité et confiance avec les enquêtés à qui nous donnions des nouvelles des autres membres de la famille éloignée. À Ghoumrassen, des entretiens ont été effectués auprès des anciens maire et directeur d'école, afin d'éclairer certaines évolutions socio-économiques de la ville; et à Marseille, auprès d'un agent de la Préfecture des Bouches-du-Rhône pour comprendre les évolutions de la procédure du regroupement familial. La connaissance ethnographique des sites s'appuie sur des terrains revisités de longue date par les auteurs (Belsunce, Ghoumrassen) non dans le but d'éprouver la solidité de résultats passés [Burawoy, 2003], mais à l'inverse, afin d'y mesurer les modifications en cours. Enfin, l'écriture à quatre mains ne signifie pas ici la tentative de croisement de deux regards partiels sur une réalité considérée comme éclatée ou antagoniste, mais au contraire, le croisement de deux approches scientifiques, qui s'ignorent souvent, sur une réalité pensée comme unitaire. L'objectif de la démarche n'est pas d'opposer une sociologie de l'intégration à une sociologie des migrations, mais bien de croiser les deux approches pour mieux saisir les formes sociales d'un processus migratoire.

\section{Une histoire familiale dans l'Histoire de l'immigration maghrébine}

L'histoire de la famille C. est par certains aspects exemplaire des grandes étapes de l'histoire sociale de l'immigration maghrébine en France: depuis les pionniers venus du milieu rural salariés dans les usines de grandes villes françaises dès les années 1950 - jusqu'à leurs enfants arrivés en nombre dans les années 1980, et leurs petits enfants insérés dans l'activité commerciale depuis les années 2000. Mais par certains autres aspects, elle s'en différencie, du fait même qu'il s'agit de carrières d'immigrés vite reconvertis dans le commerce, et dont l'histoire sociale en France de l'évasion ouvrière vers le petit commerce et l'artisanat est méconnue ${ }^{2}$.

Il s'agit d'un clan familial dont une partie des membres, immigrés à Paris [Boubakri, 1985], se reconvertit assez tôt dans un secteur d'activité, la pâtisserie, déjà pratiqué en Tunisie dès le début des années 1950, puis en Algérie. Si certains d'entre eux, les pionniers, arrivés au milieu des années

2. Dans une étude publiée en 1977 sur l'accès à la petite entreprise familiale et artisanale par le monde ouvrier, Nonna Mayer (dont l'analyse porte majoritairement sur d'anciens ouvriers français) note cependant que les petits patrons étrangers sont plus souvent que les français d'anciens ouvriers. 73\% d'entre eux étaient ouvriers en 1965 [p. 44] 
1950, ont commencé par être embauchés en France dans les secteurs du Bâtiment et des travaux publics et de l'industrie alors en plein essor, la majorité d'entre eux, venus par la suite en 1960, a travaillé directement dans un commerce alimentaire (pâtisserie orientale, épicerie, restauration) tenu en association avec des membres du clan déjà installés.

Aux premiers temps de leur activité commerçante en France, les «pères fondateurs » sont associés entre frères ou cousins dans des établissements gérés conjointement. La norme familiale, en vigueur dès la création de pâtisseries à Ghoumrassen ${ }^{3}$, et qui implique que les membres d'une même fratrie soient toujours associés dans chaque nouvelle affaire, est adaptée au contexte migratoire. Un système solidaire de gestion commerciale et financière par alternance [Boubakri, 2002] est mis en place, servant non seulement au développement et à l'extension du réseau commercial contrôlé par le groupe, mais aussi au soutien économique et éducatif des familles (femmes et enfants) restées à Ghoumrassen ou à Tunis. Il permet à ces commerçants de Marseille des absences prolongées pour des séjours fréquents dans la ville d'origine et à Tunis (deux ou trois fois par an, pour une durée de trois à six mois). Il a longtemps rendu possible le choix d'une double présence familiale et économique entre, d'un côté, la France, où sont localisées les affaires principales de la famille (commerces alimentaires, restauration, bazars, etc.) et, de l'autre, la Tunisie où demeure tout ou partie de la famille, et où elle contrôle d'autres affaires (commerce, hôtellerie, exploitations agricoles, imprimerie).

L'ouverture d'une première pâtisserie à Marseille date de 1957. Haj M'hemed, Moktar, Salem (puis plus tard R'houma) créent alors, en association, les premiers établissements de la famille C. dans le quartier Belsunce, au cœur de la ville. Un peu plus tard, au milieu des années 1960, le deuxième groupe de cousins, composé des frères Mohcen, M'hemed et Belgacem, ouvre une deuxième pâtisserie, en association avec eux. C'est ce que précise R'houma :

«Je suis né le 7 août 1945 à Ghoumrassen, où j'ai été à l'école jusqu'à 1960. En 1962, j'ai été en Algérie où j'ai rejoint mon frère Salem qui avait une épicerie, jusqu'en 1965. Après l'indépendance de l'Algérie, je suis venu à Marseille travailler avec mes deux autres frères M'hemed et Moktar qui venaient d'ouvrir une deuxième pâtisserie en association avec mes cousins. »

Depuis l'âge de 17 ans, R'houma travaille en association avec ses frères. Avant son mariage, il a toujours réservé une partie de ses revenus, soit pour apporter une aide financière aux épouses et enfants de ses frères mariés restés à Ghoumrassen, soit pour participer à l'achat de fonds de commerce. Quand bien plus tard, proche de la retraite, il décide de rentrer à Ghoumrassen pour s'occuper des terres familiales, ses frères lui remboursent leur « dette passée » en lui laissant tout l'argent de la revente d'un fonds de commerce.

Ces frères et cousins connaîtront à Marseille, comme d'autres commerçants immigrés du centre-ville populaire, les années fastes de la décennie 1970 (jusqu'au milieu des années 1980), et celles noires de la période 1985-1995, avec un tourisme maghrébin ralenti par des restrictions de sortie du territoire liées à l'obtention du visa français (Règle instaurée en septembre 1986) et de sortie de devises imposées par les pays du Maghreb (mars 1986) [Tarrius, 1987], conjugué aux retombées de la guerre civile en Algérie et à celles d'une politique urbaine de réhabilitation associant de fréquents contrôles policiers dans les hôtels, petits restaurants et marchés du secteur [Mazzella, 1996].

Dans ce contexte, au milieu des années 1980, l'activité professionnelle des deux fratries de la famille C. se diversifie aussi bien en Tunisie qu'en France. En Tunisie, les investissements des membres de la famille se font dans l'hôtellerie, le secteur productif (imprimerie) et agricole ; tandis qu'en France, ils concernent le commerce de bazar et de la restauration rapide (snacks). Aujourd'hui, leur activité commerciale est principalement implantée à Marseille : dans les premiers quartiers d'installation proches de la gare et du port de la ville, tels que Belsunce et Noailles, et depuis 2000 dans des secteurs situés plus au Nord de la ville, vers la Joliette, ou plus au Sud, dans

3. Rattaché administrativement au gouvernorat de Tataouine, Ghoumrassen constitue une municipalité de plus de 11000 habitants. La ville est le chef-lieu de la délégation du même nom qui compte 18335 habitants qui rassemble, outre la ville de Ghoumrassen, les villages de Ksar Hadada, Elferch, Tlalet, Elhorria, Ksar Elmorabitin et Graguer. Elle fait partie de ces régions rurales du Sud tunisien qui ont connu dès les années 1970 une forte émigration internationale vers la France en particulier [Baduel, 1977]. 
les rues de l'hypercentre commercial, telle la rue de Rome qui fait partie des quatre grands axes commerciaux du centre-ville de Marseille (carte 1).

Carte $\mathrm{n}^{\circ} 1$. La ventilation des commerces de la famille C. dans le centre-ville de Marseille

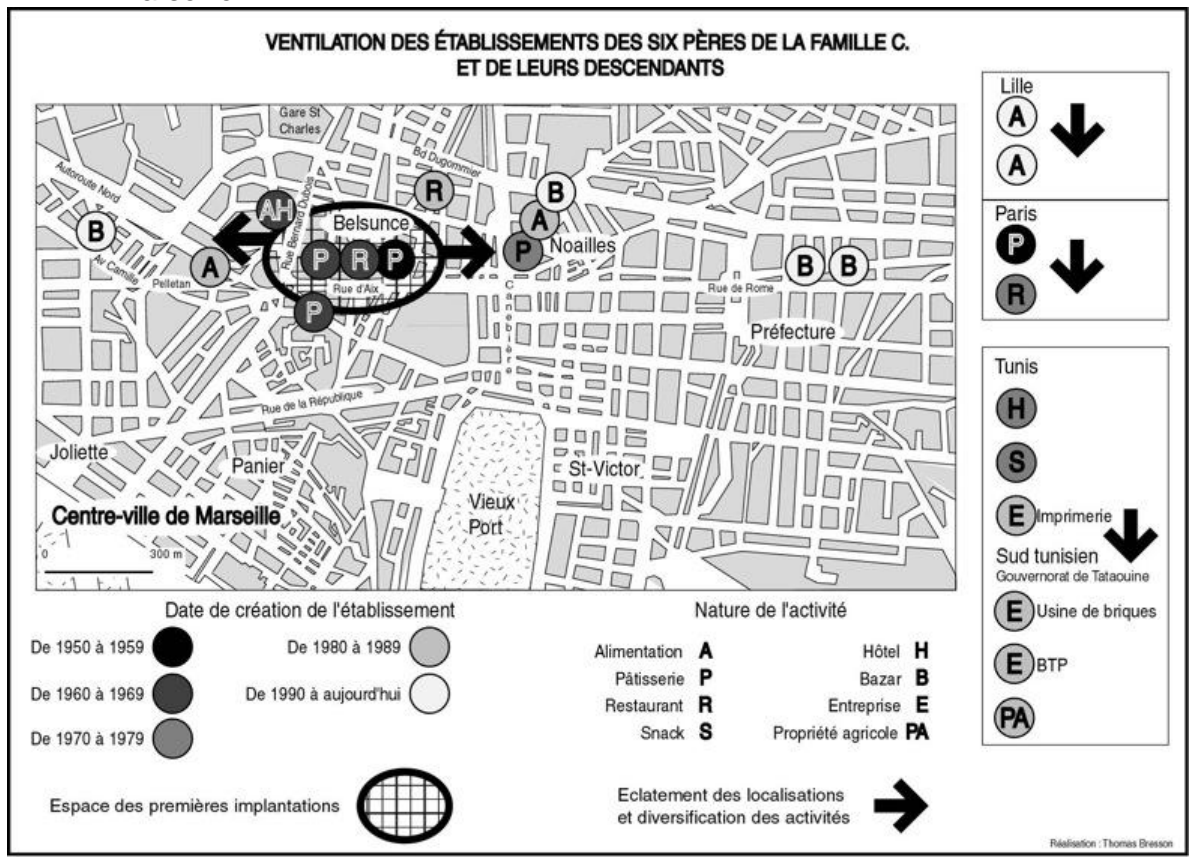

\section{Le regroupement familial à l'échelle de la famille transnationale élargie}

Le regroupement familial s'inscrit à différents moments du parcours migratoire du clan C. Lorsque la question du regroupement familial est subsumée par les transmissions entre les parents et les enfants, entre les filles et les garçons, mais aussi entre les générations de migrants, le changement d'échelle, de la famille nucléaire à la famille élargie, permet de mieux mesurer les contraintes communautaires qui pèsent sur les négociations et les décisions du père et de la mère.

La plupart des pères de la génération pionnière sont venus dans un premier temps seuls rejoindre, qui un frère, qui un cousin, ou un proche. Les femmes et les enfants en bas âge sont restés dans la région natale. Certains leaders du groupe familial, à l'instar de Mohcen, ont été rejoints par leurs familles dès la fin des années 1960. Des ménages se sont ainsi installés à Paris et à Marseille, donnant naissance à une «deuxième génération » qui a aujourd'hui plus de quarante et parmi laquelle certains ont acquis la nationalité française. À l'époque cependant, certains pères arrêtent ou interrompent la procédure de regroupement familial engagée, ce qui souligne le caractère pionnier de ceux qui ont osé prendre une telle initiative perçue par les autres membres du groupe comme une entorse aux conventions sociales, voire une trahison de la famille et de la ville natale ${ }^{4}$.

\footnotetext{
${ }^{4}$. A. Sayad [1999, p. 56] rappelle que : «Pendant longtemps, alors même que l'immigration familiale pouvait être désirée (individuellement) par l'immigré et par son épouse, qui n'ignoraient pas qu'ils s'exposaient de la sorte à
} 
Pour la majorité de ces pères de famille, le recours à la procédure du regroupement familial se fait donc plus tardivement et de manière partielle. Il faut attendre le milieu des années 1980, pour que leurs fils, âgés de 13 à 18 ans $^{5}$, viennent les rejoindre à Marseille ${ }^{6}$. Ces adolescents sont les seuls concernés jusqu'en 1993, date à partir de laquelle la loi n'autorise plus le regroupement partiel, mais prévoit le regroupement de l'ensemble de la famille (femme et enfants mineurs).

Une loi évolutive
La rédaction des articles 29 et 30 bis de l'ordonnance de 1945, relatifs au
regroupement familial, laisse à penser que faire venir sa famille est un droit dont
l'exercice ne suscite aucune difficulté majeure. L'objectif affiché de la loi de 1993 sur
le regroupement familial est d'assurer : « une intégration réelle en créant un véritable
statut du regroupement familial [...] en garantissant l'insertion paisible et durable des
familles » (selon Charles Pasqua, alors ministre de l'Intérieur). Avec l'adoption des
dernières lois relatives à l'immigration en France du 24 juillet 2006 et du 20 novembre
2007, les conditions d'admission au titre du regroupement familial se durcissent. Un
étranger ne peut solliciter un regroupement familial qu'après dix-huit mois de séjour en
France, contre douze précédemment. Il doit prouver qu'il peut subvenir aux besoins
des siens par son seul travail et les loger de façon convenable. Le regroupement
familial est refusé si le bénéficiaire ne respecte pas "les principes qui régissent la
République française », en particulier en cas de polygamie. Les préfets ont la faculté de
retirer le titre de séjour d'un conjoint admis en France au titre du regroupement
familial «en cas de rupture de la vie commune ». Une évaluation du degré de
connaissance de la langue française et des valeurs de la République est faite. La
commission du Sénat supprime le 26 septembre 2007 l'article 13 très controversé en
France du projet de loi Hortefeux, qui autorisait à titre expérimental jusqu'au 31
décembre 2010 le recours à des tests ADN sur la base du volontariat pour les étrangers
candidats au regroupement familial.

Les mères de famille sont loin d'être passives dans le maintien de l'unité familiale entre plusieurs territoires. Ces mères « voyageuses ", âgées aujourd'hui de plus de cinquante ans, certes minoritaires dans la configuration familiale élargie, justifient leur mobilité entre Marseille, Tunis et Ghoumrassen par la nécessité d'assurer un équilibre familial dans l'éducation des enfants et dans la gestion des patrimoines familiaux en différents lieux. Cela contraint les deux membres du couple à se relayer dans cette tâche de "présence rotative».

La nouveauté résiderait moins dans la découverte de la mobilité de ces mères de famille ${ }^{7}$ que dans les effets inattendus du regroupement familial sur leur mobilité pendulaire. Depuis que la procédure administrative de regroupement familial au profit des enfants mineurs demande que ces derniers soient accompagnés de leurs mères ${ }^{8}$, le départ en France de femmes âgées de 50 à 65 ans est devenu plus fréquent. Une fois le titre de séjour obtenu (visa D, regroupement de famille, valable un an ou dix ans selon le titre de séjour du mari), ces mères de famille font la navette entre les deux pays, deux à trois fois par an, pour demeurer auprès des membres de la famille résidant de part et d'autre de la Méditerranée. Dans le cas où les mères n'ont que des fils, elles choisissent pour la majorité d'entre elles de partir en France rejoindre leur mari. Lorsqu'elles ont des filles, le choix

enfreindre la règle communautaire et à manquer à la morale du groupe, elle était effectuée et surtout elle était ressentie comme un acte honteux, un acte qu'on avait soin de cacher au point de devoir quitter le village nuitamment. »

5. Dix-huit ans est l'âge limite qui donne droit aux descendants d'un migrant, résidant régulièrement en France, de rejoindre leur ascendant par la voie du regroupement familial.

6. L'agent de la Préfecture des Bouches-du-Rhône, en charge du regroupement familial au service des étrangers, traite en moyenne, par an, moins de 150 dossiers de demande de regroupement de Tunisiens de France pour un total, toutes nationalités confondues, de 1000 dossiers en moyenne par an. (entretien, mai 2008).

7. Nancy Green [2002 ; p. 107] souligne que la redécouverte d'un passé mobile des femmes est autant une nouveauté historique que le résultat de questionnements historiographiques innovateurs permettant de réviser, à la fois, l'histoire des femmes et celle des migrations.

8. Pour l'un des anciens maires de Ghoumrassen interrogé sur la situation migratoire de sa petite ville « [...] la loi Pasqua de 1993 est à l'origine du drame de Ghoumrassen. Cette loi est en train de vider la ville de ses habitants ». (Entretien, avril 2005) 
de partir rejoindre leur mari est plus difficile. La mère peut décider de rester avec ses filles et ses plus jeunes garçons à Ghoumrassen ou à Tunis, quand le père s'occupe des aînés en France.

Ces mères de famille contribuent à maintenir l'unité familiale dans des configurations où la fratrie est le plus souvent séparée : il n'est pas rare de rencontrer des cas où les filles, mineures ou majeures, restent en Tunisie quand leurs parents sont en France accompagnés des fils. Mais cela ne se vit pas sans tensions internes dans la famille nucléaire. C'est le cas de la famille de Belgacem. Belgacem a décidé de faire venir sa femme et tous ses fils en France, alors qu'il s'y était pris trop tard pour ses filles (elles ont plus de 18 ans). Ses quatre garçons sont en France, la mère est venue par la procédure du regroupement familial s'occuper des deux plus jeunes garçons, alors que ses deux filles aînées sont inscrites dans des lycées privés à Tunis.

Comment expliquer que Belgacem n'ait pas procédé à une demande de regroupement familial pour ses filles quand tout le monde sait, à Ghoumrassen, qu'il faut l'effectuer avant les 18 ans des enfants? Il semble avoir changé d'avis sur le projet migratoire de ses filles. Il espérait qu'elles finissent leur scolarité en Tunisie. Aujourd'hui, le couple, surtout sa femme, vit mal cette situation. Son épouse menace de retourner vivre définitivement en Tunisie avec ses deux derniers fils, afin de rejoindre ses filles, si la situation familiale ne change pas. Elle lui reproche de ne pas avoir fait les démarches administratives nécessaires à la venue de ses filles. Elle nous confie mal supporter également les reproches acerbes de sa belle-sœur et de ses cousines sur le fait de laisser seules ses filles en Tunisie. De leur côté, les jeunes filles interrogées à Tunis, disent ne pas avoir encouragé leur père dans cette démarche de regroupement familial. Elles disent préférer rester à Tunis, où elles ont leurs amis, leurs habitudes, et se disent plus rassurées à l'idée d'y poursuivre leurs études.

\section{Diverses mobilités pendulaires au sein de la famille élargie}

Si les pères pionniers ont vécu depuis les années 1960 une destinée migratoire comparable ponctuée d'étapes communes, leur mobilité spatiale actuelle rend compte de choix professionnels et résidentiels relativement divergents. Au-delà des homologies évidentes de trajectoires, que nous apprennent ces récits de vie sur les espaces vécus de la migration ? L'analyse de la mobilité spatiale des différents pères de famille (durée et fréquence de leur séjour dans les trois sites d'enquête), souligne que les hommes de cette première génération travaillent à tenir le lien à la fois ici et là-bas, dans un souci de «double présence»: non seulement au maintien du lien familial intergénérationnel, mais aussi au maintien du lien économique entre des localités transnationales (carte 2).

Carte 2 - La répartition des espaces vécus des pères de famille

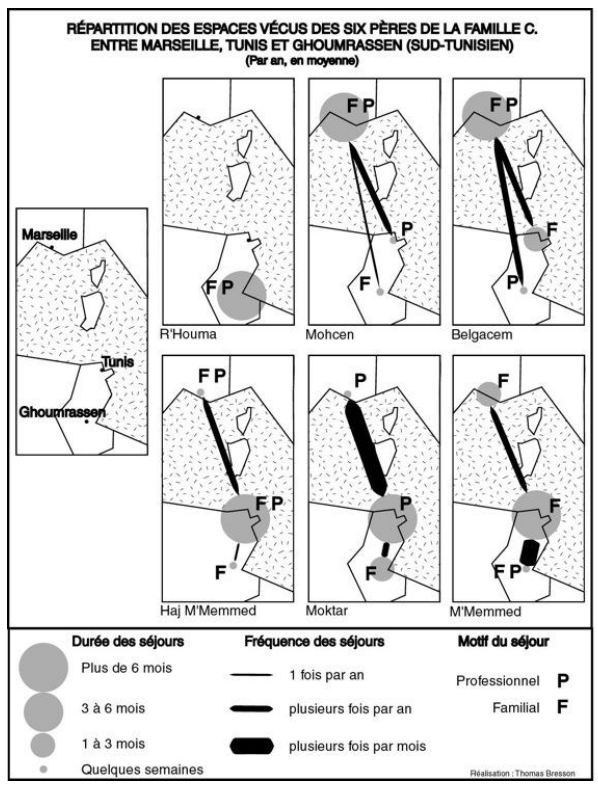




\section{Vivre et travailler à Ghoumrassen}

« Chacun choisit sa vie », nous dit sereinement R'houma, puis il ajoute: «Mes frères ont choisi le commerce, moi, j'ai choisi l'agriculture. J'ai réussi, je suis tranquille, j'ai la santé. Je ne gagne pas beaucoup d'argent mais j'ai beaucoup de terre et bientôt des olives ».

L'investissement dans la terre, par les immigrés, n'est pas un phénomène nouveau. Des études [Bencherifa, 1993; Boubakri, 1985] ont souligné que de nombreux immigrés avaient repris conscience de la valeur de la terre et des profits qu'ils pouvaient en tirer au moyen de techniques perfectionnées telles que le goutte-à-goutte. À Ghoumrassen, la culture irriguée s'est développée, à la fin des années 1980, avec l'aide de l'État, qui encourageait à cette époque sa gestion par des associations. R'houma Ben Mohamed Ben Salem (frère de Salem, Haj M'hemed et Mokhtar) est la personne qui gère, depuis 1988 , la propriété agricole familiale située à Ghordab (à l'est de Ghoumrassen), une terre de plusieurs hectares regroupant trente-trois propriétaires. Principal interlocuteur sur place du ministère de l'Agriculture, il est le président de l'association locale pour la gestion de l'eau d'irrigation, dont le bureau est composé notamment de son frère Mohktar (trésorier) et de son cousin M'hemed (secrétaire). R'houma y est propriétaire de vingt-cinq hectares; Moktar et ses deux cousins M'hemed et Belgacem y possédent également quelques hectares.

À chaque nouvel entretien, R'houma nous accueille chaleureusement, dans le gîte aménagé sur ses terres qu'il habite, chaque été, avec sa femme ou, plus tard, chez lui, entouré de sa femme et de ses quatre enfants restés à Ghoumrassen : deux filles de 13 et 14 ans, un garçon de 9 ans, ainsi que sa fille Hazar mariée, diplômée du supérieur, qui attend une réponse à sa demande de regroupement familial afin de rejoindre son époux à Marseille. Son fils aîné est en France, parti quatre ans auparavant clandestinement, sur " un coup de tête » avec deux amis d'enfance, et ce malgré la forte opposition du père à tout départ à l'étranger. Son fils travaille aujourd'hui légalement à Marseille comme vendeur dans l'établissement de son oncle Moktar.

$\mathrm{R}$ 'houma se déplace rarement en dehors de sa région sauf pour voir son fils à Marseille. Il est le seul parmi «les pionniers » à avoir clairement décidé d'arrêter le commerce et à rentrer vivre et travailler à Ghoumrassen, afin de s'occuper de ses parents âgés, de ses enfants, et de la terre. Il retire des dividendes des parts d'une imprimerie achetée à Tunis avec ses frères. On peut supposer, à l'écouter, qu'il a sans doute été déçu de sa mise à l'écart par ses frères dans l'achat d'un hôtel situé dans le centre de Tunis, et que cela a sans doute contribué à sa décision de se retirer sur ses terres à Ghoumrassen.

\section{Vivre et travailler à Tunis, et se déplacer entre Marseille et Ghoumrassen}

Un autre cas de figure réunit Moktar, Hadj M'hemed et M'hemed. Moktar est le seul parmi les six frères et cousins à ne pas vivre, la majeure partie de son temps, avec son épouse. Il a gardé un même mode migratoire. Il continue de rester trois à six mois près de sa femme et partage son temps professionnel entre Tunis, où il gère plus de six mois par an un hôtel avec ses fils, et Marseille, où il se rend fréquemment, plusieurs fois par an, et où il maintient une activité commerçante intense. Sa femme vit à Ghoumrassen sans jamais se déplacer en France ni même à Tunis. Son frère Haj M'hemed est moins présent que lui dans la gestion des affaires à Marseille. Il délègue cette charge à Moktar. Il vit à Tunis avec sa femme et ses deux filles. Il se déplace à Marseille, accompagné de sa femme qui a un visa $\mathrm{D}$, durant quelques semaines dans l'année pour rendre visite à ses deux fils ânés commerçants. Il réserve toujours un mois de l'été pour se rendre à Ghoumrassen. Son cousin M'hemed vit également avec sa femme et ses filles à Tunis. Ses voyages Ghoumrassen sont plus fréquents que ceux de ses frères et cousins. Cette fréquence, plusieurs fois par an, s'explique par son implication dans la gestion de la terre de Ghordab avec son cousin R'houma. Il se rend seul à Marseille (son épouse n'a pas de visa D), principalement pour rendre visite à son fils Chokrem parti depuis dès l'âge de 16 ans, et qui travaille maintenant en association avec son cousin Zahreddine. 


\section{Vivre et travailler à Marseille, et se déplacer vers Tunis et Ghoumrassen}

Cette mobilité pendulaire est illustrée par Mohcen et Belgacem. Mohcen est l'un des premiers qui, dès 1966, prend la décision de venir vivre en France avec sa femme et ses deux fils. Il quitte régulièrement Marseille, plusieurs fois par an, pour se rendre quelques semaines à Tunis dans le but de suivre les affaires commerciales et, une fois par an, à Ghoumrassen où durant l'été, il retrouve le reste de la famille élargie. Sa femme l'accompagne régulièrement dans ses déplacements.

Belgacem, est de tous les enquêtés celui qui semble le plus mal à l'aise dans ce choix de vie qui le partage entre la France et la Tunisie. La situation familiale éclatée mal vécue crée des tensions au sein du couple, et entre les parents et les filles aînées restées à Tunis. Plusieurs fois par an, Belgacem, en alternance avec sa femme, effectue de longs séjours de plusieurs mois dans la capitale tunisienne. C'est la solution provisoire que le couple a trouvé de façon à assurer une présence parentale quasi constante auprès des filles aînées. Par ailleurs, Belgacem se rend seul deux fois par an à Ghoumrassen pour s'occuper de ses terres cultivées.

\section{L'impact d'une expérience transnationale sur la seconde génération}

Dans cette dernière partie, nous interrogeons le poids de la destinée familiale et en retour, les arrangements que les enfants trouvent pour composer avec elle. Il ressort que ce n'est pas un modèle de destinée héritée «tel père tel fils » qui prévaut de manière mécanique, mais que les revendications des enfants suscitent de nouveaux compromis nécessaires à la pérennisation même de l'unité de la famille transnationale.

\section{La migration pour diplôme chez les garçons}

La génération des fils, âgée aujourd'hui, entre 15 et 45 ans, a globalement échoué dans sa scolarité à Ghoumrassen. Pour la grande majorité d'entre eux, l'échec scolaire veut être compensé par la réussite sociale du projet migratoire. Le garçon adolescent prend conscience très tôt qu'il sera un jour ou l'autre envoyé en France dans la famille commerçante, proche des frères ou du père. Aussi, dans les récits, il est constaté une perte précoce de motivation des garçons pour les études générales ou professionnelles, qui n'intéressent pas ces fils destinés à être commerçants. Le récit de Chokrem sur son parcours illustre combien l'aspiration au départ à l'étranger l'emporte sur la réussite scolaire avant même l'adolescence. Cela nous a été aussi confirmé lors d'un entretien avec l'ancien directeur d'école de Ghoumrassen qui y a travaillé durant vingt ans, et qui a constaté durant ces années le «décrochage scolaire » précoce de ces jeunes élèves garçons, fils de commerçants émigrés.

«Les premières années, j'étais fort à l'école primaire. Suite à mon premier voyage en France à l'âge de 9 ans, je suis rentré à Ghoumrassen avec une autre idée dans la tête. Je me suis dit: "Moi, mon avenir c'est en France ! Je vais travailler". Je ne pensais plus aux études. Les études, j'ai laissé, j'ai dit : "Tôt ou tard, je vais venir en France". Mon oncle avait un commerce à Marseille et mon père à l'époque un restaurant à Paris. J'ai alors dit : "En fin de compte, les études ça va me servir à rien". Ensuite, les études ça n'a plus marché, si je n'étais pas le dernier, j'étais l'avant-dernier. J'ai alors redoublé mon année. Parce que, dans ma tête, j'étais déjà en France. J'ai dit à mon père: "Moi, ma vie c'est la France". La Tunisie, j'y pensais même pas. Et à 16 ans, j'ai arrêté les études. En plus, j'étais dans une école professionnelle. Je me suis dit: "Même si je continue, dans un an, je vais obtenir un diplôme ; CAP d'électricité, ça va me servir à quoi ? À rien parce que, dans un an, je vais aller en France pour travailler". Mon diplôme, c'était en fait de venir en France. Et voilà, depuis, je suis 
resté ici [...].» (Extrait d'entretien avec Chokrem, 31 ans, fils de M'hemed C, exploitant d'un commerce de bazar, rue de Rome à Marseille)

\section{La sortie du modèle commercial d'alternance des pères}

Si la solidarité familiale dans la migration continue de jouer en faveur de ces jeunes commerçants, il ressort de l'analyse des cas de création ou d'achat de nouveaux commerces par des fils, en quelque sorte affranchis de l'autorité de leurs aînés (pères ou oncles). Ces jeunes choisissent par exemple de s'associer avec des membres de la famille de leur génération, d'encourager la création de commerces dans de nouveaux secteurs d'activité, et vont jusqu'à remettre en question le modèle de gestion en alternance.

Zahreddine, fils de Mohcen et cousin de Chokrem, est en France depuis l'âge de 9 ans. Il nous explique les longues tractations passées avec ses oncles (Belgacem et M'hemed) et son père, pour aboutir finalement, sans conflits, à l'ouverture d'un commerce de bazar géré par lui en relative autonomie $^{9}$. Chacune de ses aventures commerciales, de la pâtisserie au bazar, traduit la quête d'une plus grande distanciation vis-à-vis du modèle parental traditionnel de gestion commerciale et d'implication de la famille élargie. Cette demande d'autonomie commerciale et financière, vis-à-vis des oncles et cousins, est d'autant mieux acceptée que le partage est considéré comme équitable, et que la relation de travail mêlant le familial à l'économique n'est pas rompue, mais seulement distendue entre ses membres.

«Moi, au début, je faisais des propositions et donnais des idées, mais comme j'étais jeune, mon père et mes deux oncles ne m'écoutaient pas. Un jour j'ai dit : "Pourquoi ne pas partager. Chacun prendrait un magasin ou un local, chacun partirait tout seul, chacun ferait à son goût". Mon oncle M'hemed était un peu d'accord, mon oncle Belgacem n'était pas d'accord, mon père était mitigé, parce qu'il y avait quand même une histoire de famille et il ne voulait pas se séparer de ses deux frères. Je trouvais pourtant que ça pouvait faire avancer les choses, mais en même temps, à l'époque, je trouvais aussi que ça ne me regardait pas. Ce qui me gênait, c'est que cela me freinait. Moi je voulais aller beaucoup plus loin que ça. Je ne voulais plus recevoir d'ordres. On n'avait pas la même idée commerciale. Je voulais travailler une année encore dans la pâtisserie, et repartir encore vers autre chose. Mais eux ne voulaient pas réinvestir l'argent et créer d'autres branches [...]. Mon idée s'est réalisée finalement, mais après plusieurs affaires échouées avec mes oncles et mon père et beaucoup d'énergie de ma part. J'ai finalement fait grandir la société en ouvrant d'autres magasins de bazar pour qu'on me laisse tranquille. Tout le monde, maintenant, gère son propre magasin à sa façon. » (Extrait d'entretien avec Zahreddine, 45 ans, gérant d'un commerce de bazar dans le centre ville de Marseille, rue Noailles).

Quand ces formes d'individuation n'aboutissent pas à des ruptures, elles soulignent, au contraire, des formes de négociation au sein de la famille et nous donnent la mesure de sa capacité à se recomposer dans la dynamique des générations. Les cas de rupture familiale mentionnés sont de jeunes garçons de la famille (le fils cadet de M'hemed, et celui de Rhouma parti en France bien que son père ait caché son passeport) et ont conduit au moins un temps l'individu à la clandestinité, coupé de toutes ressources du clan.

\section{Le choix des études des filles : une émancipation sous contrôle}

Si les garçons enquêtés misent dès leur plus jeune âge sur le départ à l'étranger, les filles misent davantage sur la scolarité comme voie de promotion sociale, et sont soutenues en cela financièrement et moralement par leur famille. Gouta et Ridha, filles de M'hemed, sont de jeunes

9. Zahreddine obtiendra le fonds du nouveau commerce à son nom le jour où il aura remboursé, sans frais, le capital prêté par ses parents et oncles. Jusque-là en tant que gérant du fonds, il ne reçoit que le tiers des bénéfices. S'il veut devenir associé (au tiers par exemple), il devra rembourser le tiers du capital initial investi par les propriétaires réels, ou bien la moitié, s'il veut être associé à la moitié. 
filles bachelières qui ont préféré rester en Tunisie pour mener à terme leurs études supérieures à Tunis. Lors d'entretiens réalisés à Tunis, elles déclarent ne pas être intéressées par l'émigration «pour le moment». Si Ridha envisage d'aller en France pour les vacances, Gouta n'y songe même pas. Moufda, leur sœur cadette, a déjà demandé à leur père de lui préparer les papiers pour le regroupement familial, comme il l'a fait pour son fils Saïd. Mais, selon elles, le père n'acceptera pas que sa fille cadette parte. Leur frère Chokrem, depuis Marseille encourage Ridha, qui affiche un goût affirmé pour les études, à poursuivre ses études supérieures. C'est toujours lui qui convainc ses parents de la laisser quitter Ghoumrassen pour Tunis, afin que Ridha puisse s'inscrire à l'université. Ses parents ne voulaient pas qu'elle aille seule en cité universitaire. Sa mère et ses sœurs ont alors trouvé un compromis : elles ont décidé de quitter Ghoumrassen pour vivre à Tunis. Cela a aussi permis aux sœurs cadettes, Gouta et Moufda, d'aller dans des lycées et collèges privés qui n'existaient pas à Ghoumrassen.

D'autres cas de figures relevés concernent de jeunes femmes qui décident de rejoindre leur mari en France, après l'obtention d'un diplôme supérieur acquis en Tunisie. Elles partent en tant que futures doctorantes continuer un troisième cycle, ou comme employées salariées dans le réseau commercial de la famille. Contrairement à leurs mères qui n'ont pas poursuivi d'études secondaires dans la plupart des cas, et ont connu tardivement une mobilité vécue comme contrainte, ces jeunes filles restent déterminées à poursuivre leurs formations ou leurs carrières professionnelles dans le projet migratoire décidé avec leurs maris. C'est une piste de réflexion qui reste à explorer pour la compréhension de formes d'individuation sexuée au sein de la famille transnationale. Elle souligne des formes de participation des jeunes femmes diplômées dans la prise de décision, avec leur conjoint, du regroupement familial, et dans la gestion des affaires familiales.

Les autres cas rencontrés de jeunes filles célibataires qui ont fait le choix de poursuivre des études supérieures en France, ont conscience de certaines attentes fortes de la famille élargie. Elles ont du mal à imaginer la possibilité de se marier avec une personne hors du groupe de parenté de la famille élargie. C'est ce que confie l'une d'entre elles, Hayet, la fille de Moktar, étudiante de 22 ans en Master à l'Université de Nice depuis deux ans :

«Nos parents se sacrifient pour nous en se saignant pour nous payer les études. On représente tout pour eux, nous ne pouvons pas les décevoir en nous mariant hors du groupe. Ils ne nous disent pas : "Je t'interdis de le faire !"; mais ils nous répètent : "Je te fais confiance !", et c'est pire. »

L'éloignement du foyer familial et par conséquent l'horizon des possibles qu'ouvre la migration sont vécus chez ces jeunes filles comme une épreuve de fidélité aux valeurs familiales. Elles éprouvent un sentiment de dette envers leur famille sur le leitmotiv : «On ne peut pas les décevoir ». Ici le sentiment de dette morale envers leurs parents éloignés rappelle ce que souligne Nina Glick-Schiller [2004, 2006] à propos du fonctionnement du champ social transnational qui repose à la fois sur des façons d'être (ways of being) et des façons d'appartenir à distance (ways of belonging): considérant d'un côté les relations et les pratiques sociales transnationales par lesquelles s'engagent et se lient les individus, et de l'autre, la mémoire, la nostalgie, ou encore l'imaginaire (par exemple chez les jeunes garçons du groupe celui de la France qui l'emporte sur la réalité des études) qui nourrissent une co-présence à distance. Le mariage endogame (avec un musulman du groupe de parenté) se perpétuerait dans ce cas hors frontières au sein des jeunes générations, en particulier chez les jeunes filles parties seules étudier, mais sans que cela prenne la forme d'un interdit ${ }^{10}$.

Le terrain d'enquête ne permet pas d'approfondir ce questionnement du mariage endogame, objet d'un champ d'analyse en anthropologie et en droit, qui aurait mérité en soi un large

10. Comparée à l'Algérie et au Maroc, la Tunisie a assoupli sa loi concernant la pratique du mariage des femmes avec un non musulman. L'évolution du statut personnel de la femme entreprise par le président Bourguiba [Charfi, 1998], accentuée par de nouvelles interprétations jurisprudentielles de la loi tunisienne (juin 1999), fait du code tunisien de la famille un exemple caractéristique des changements actuellement soutenus par les organisations onusiennes dans la modernisation des lois internes des pays arabes conventionnellement engagés. Le code de la famille contrairement à celui des autres pays arabes a gardé le silence sur des questions essentielles telles que la validité du mariage d'une musulmane avec un non musulman, ou la successibilité entre musulmans et non musulman. Ces questions ont été laissées au pouvoir d'interprétation du juge. 
développement. Des travaux ont cependant souligné que la recomposition de l'espace de parenté dans l'immigration perpétue une pratique de l'endogamie familiale sans qu'elle soit pour autant ressentie comme une clôture, un isolat culturel ou une reproduction de la tradition [Streiff-Fénart, 1999 ; Rude Antoine, 1999]. Cette pratique est d'autant plus acceptée par les plus jeunes qu'elle ne repose pas sur un strict interdit. Le principe de l'endogamie est une norme contraignante qui est la condition même de l'existence du groupe en migration. Mais cette condition sine qua non bien que structurante n'est pas exclusive de dynamiques sociales à l'œuvre entre les générations, entre les sexes, entre les territoires investis nécessaires à la reconfiguration de la famille transnationale.

\section{Conclusion}

La question du regroupement familial s'inscrit d'ordinaire dans l'analyse juridico-politique de la notion d'intégration et de la capacité de l'État-nation à enraciner les populations étrangères qui travaillent depuis longtemps sur son sol. En partant du berceau rural où s'est forgé le projet migratoire d'une famille tunisienne, il s'est agi ici de renverser la perspective : comment les membres de cette famille intègrent les contraintes légales françaises pour construire par le bas, d'une génération à l'autre, un champ familial transnational, entendu comme un système structuré et structurant de dispositions durables et transformables entre différents contextes nationaux. La question du regroupement n'étant plus seulement perçue comme une adaptation aux normes du pays d'installation, mais comme une des contraintes exogènes, s'ajoutant à celles endogènes, d'un projet nomade. Plus encore, l'intégration de différentes contraintes, de différentes valeurs, et de différentes expériences, d'une génération l'autre, infléchit le modèle familial et le contenu même du projet migratoire initial des aînés.

Malgré tous les obstacles et les destinées diverses des membres de la famille, des parents et des enfants, des hommes et des femmes, des ruraux et des urbains, c'est la tentative de création et de maîtrise d'un territoire familial élargi - une portion méditerranéenne de l'espace transnational entre Ghoumrassen et Marseille - que cette famille tunisienne tisse à travers deux générations.

Quand la question du regroupement familial n'est pas considérée du simple point de vue juridique français, elle peut être un critère pertinent d'analyse d'une certaine mobilité migratoire. Elle implique alors de croiser les points de vue de la France et de la Tunisie, pour saisir, de part et d'autre de la Méditerranée, les différentes pratiques du regroupement familial, suivant les stratégies de mobilité des familles. Cette question, ainsi posée, permet l'analyse des effets de l'expérience transnationale sur chacun de ses membres, hommes et femmes. Plus largement, elle éclaire quelques unes des tensions internes entre projet économique et norme sociale, entre espace commercial et territoire agricole, entre nomadisme et sédentarisme, dans la recomposition intergénérationnelle d'un espace familial transnational.

\section{Bibliographie}

BADUEL P. [1977], Les conséquences sociales de l'émigration temporaire en Europe sur la vie de la région d'origine de la délégation de Kébili (sud- tunisien), Thèse pour l'obtention du grade de docteur de 3ème cycle en sociologie, Tome 1, $137 \mathrm{p}$.

BENCHERIFA A. [1993], «Migration extérieure et développement agricole au Maroc. État de la connaissance, observations empiriques récentes et perspectives de recherches futures ", Revue de géographie du Maroc, vol. 15, $\mathrm{n}^{\circ} 1$ et 2, p. 51-92.

BENSAlah N. (dir.) [1994], Familles turques et maghrébines aujourd'hui. Évolution dans les espaces d'origine et d'immigration, Paris, Maisonneuve \& Larose, $307 \mathrm{p}$.

BoubaKRi H. [1985], Le petit commerce du Sud tunisien à Paris : espace, fonctionnement social et impact sur les régions natales, Thèse de doctorat de $3^{\mathrm{e}}$ cycle, Université de Strasbourg I, $300 \mathrm{p}$. 
BOUBAKRI H. [2002], «Les effets des réseaux transnationaux sur l'économie locale et régionale à Tataouine (Sud-Est tunisien) », in CESARI J. (dir.), La Méditerranée des réseaux, Paris, Maisonneuve \& Larose, p. 181-202.

Boubakri H,. MAzella S. [2005], «La Tunisie entre transit et immigration. Politiques migratoires et conditions d'accueil des migrants africains à Tunis », Autrepart $\mathrm{n}^{\circ} 36$, IRD/Armand Colin, 2005, p. 149-166.

BuRAwoy M. [2003], "Revisits: an outline of a theory of reflexive ethnography", American Sociological Review, vol. 68, p. 645-679.

Caglar A., Glick-SChiller N., Guldbrandsen T. [2006], "Beyond the ethnic lens: locality, globality, and born-again incorporation", American Ethnologist, vol. 33, n 4, p. 612-633.

CHARFI M. [1998], Islam et liberté, le malentendu historique, Paris, Albin Michel, 192 p.

Delcroix C., Missaoui L. [2005], «Familles, destins personnels et appartenances collectives en Migration », Revue Européenne des Migrations internationales, (introduction du dossier), vol. 21, $\mathrm{n}^{\circ} 3$, p. 7-8.

Gotman A. [1999], «Géographies familiales, migrations et générations », in Bonvalet C., Gotman A., GrafMEYer Y. (dir.), La famille et ses proches, Paris, PUF, p. 69-133

GREEN N. [2002], Repenser les migrations, Paris, PUF, 144 p.

LE GALl J. [2005], «Familles transnationales: bilan des recherches et nouvelles perspectives », Diversité urbaine, vol. 5, $\mathrm{n}^{\circ} 1, \mathrm{p}$ 29-42.

LEVITT P., GLICK-SCHILLER N. [2004], "Conceptualizing simultaneity: a transnational social field perspective on society”, International Migration Review, vol. 38, ${ }^{\circ}$ 145, p. 595-629.

MAYER N. [1977], «Une filière de mobilité ouvrière : l'accès à la petite entreprise artisanale et commerciale », Revue française de Sociologie, vol. 18, n 1, janv-mars, p. 24-45.

MAZZELLA S. [1996], L'enracinement urbain : intégration sociale et dynamique urbaine. La population maghrébine du centre-ville de Marseille, Thèse nouveau régime, sous la dir. de Jean-Claude Chamboredon, Marseille, EHESS, 400 p.

MAZZELLA S. [2008], « Marsiglia: città portuale e di immigrazione. Riflessioni sulla «seconda generazione», Mondi Migranti, n³, éd. Franco Angeli, Genova, p. 12-26.

QUiminal C. [1997], «La famille immigrée entre deux espaces », in FASSIN D., MORICE A, Quiminal C. (dir.), Les lois de l'inhospitalité, Paris, La Découverte, p. 67-80.

Rude Antoine E. [1999], «Trajectoires familiales - Transformations des rôles et statuts », in Dewitte P (dir.), L'immigration et l'intégration, Paris, La Découverte, Coll. l'État des savoirs, p. 196-204.

SAYAD A. [1999], La double absence. Des illusions aux souffrances de l'immigré, Paris, Seuil, 448 p.

STREIFF-FENART J. [1999], «Construction d'un réseau de parenté transnational : une étude de cas d'immigrés tunisiens dans le Sud de la France », Revue Européenne des Migrations Internationales, vol. $15, \mathrm{n}^{\circ} 3$, p. 45-61.

TARrius A. [1987], «L'entrée dans la ville : migrations maghrébines et recompositions des tissus urbains à Tunis et à Marseille », Revue Européenne des Migrations Internationales, vol. 3, p. 131-148. 